\title{
Rack Mounted Low-profile Indirect Frequency Converter
}

\author{
Tomas Glasberger ${ }^{1}$, Tomas Kosan ${ }^{2}$, Jan Molnar ${ }^{3}$ \\ Regional Innovation Centre for Electrical Engineering (RICE), Faculty of Electrical Engineering, \\ University of West Bohemia, Univerzitni 26, 30614 Pilsen, Czech Republic \\ E-mail: 1 tglasber@ rice.zcu.cz, ${ }^{2}$ kosan@ rice.zcu.cz, ${ }^{3}$ jmolnar@ rice.zcu.cz
}

\begin{abstract}
A new indirect frequency converter is introduced in this paper. The main goal of the new proposal is to simplify installation and running as supply unit for industrial applications. It fulfills minimum dimensions requirement and also enables parallel connection and full energy recuperation mode. The proper function of the designed converter has been performed on a prototype of linear actuator for a 6DOF motion platform.
\end{abstract}

Keywords-DSP; FPGA; frequency converter

\section{INTRODUCTION}

Electric drives for special manipulators require extraordinary approach to design of their basic parts as power semiconductor converter, electric machine and control system. The proposed converter represent a new design with requirements as low dimensions, scalability, simple enhancement and modularity including a sophisticated control system with important peripheral interfaces and significant computational power for implementation of complex control algorithms.

Scalability and modularity are important requirements on indirect frequency converters nowadays. Usually they are fulfilled with a topology where an input rectifier and an output inverter are realized as separate stand-alone modules as well as even input filter can be in form of a separated module. Energy recuperation is usually not possible because of the input diode rectifier. It is possible to share the energy among several inverters via the dc-link circuit as shown in a multi-motor system [1].

Another way to design the converter is to use just one complete unit which integrates the input filter, the input rectifier and the output inverter.

In this case, it is very advantageous to use an voltge source active rectifier called also the active front end (AFE) to support energy recuperation and its transfer between several converters connected in parallel [2]. It also enables low total harmonic distortion of the grid current.

Single converter approach can be easily installed, power enhancement can be performed using parallel connection of several converter units and it is easily replaceable in case of failure. Serial assembling is also easier if the input part is composed of the same topology (or power semiconductor module) as the output

The research has been supported by TACR under project No. TE02000103 (CIDAM) and by proiect SGS-2018-009. part of the converter. This can significantly reduce manufacturing and also control software development costs.

The proposed converter represents an universal frequency converter unit. Its first real industrial utilization is planned as a supply unit in a special six degrees of freedom motion platform/manipulator [3].

\section{MOTION PLATFORM}

As mentioned above the motion platform is the 6DOF manipulator, its schematic is shown in Fig. 1. It is composed of three rails connected to the triangle. There are placed two linear permanent magnet synchronous motors(LPMSM) on each rail (points P01-P06 in Fig. 1). These six motors enables independent moving of six rods which are connected to a load platform placed above the rails (points P11-P16) in Fig. 1).

The movement of the motors and consequently movement of the rods ensures position change of the platform e.g. horizontally and under different angles to the ground and to the vertical axis.

This configuration enables very high dynamics and precision of movement of the load platform in defined axes.

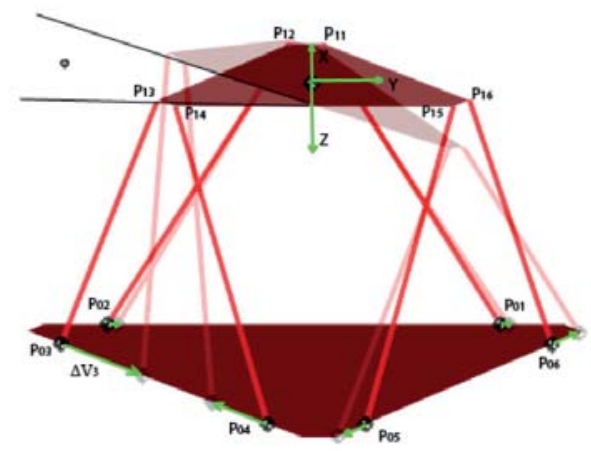

Fig. 1. Schematic of 6-DOF motion platform. [3]

To ensure high performance and dynamics of the linear motors, the designed converter and its peripherals were adopted to be utilized in this application. It can be noted that for this type of manipulator, six converters have to be used. one for each LPMSM. 


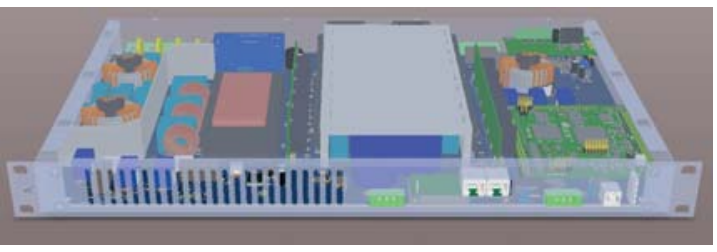

Fig. 2. 3D model of converter with enclosure

\section{MAIN BOARD}

\section{A. Power stage topology}

Power stage is designed to fit in standard 19" rack mount. There has been a demand to have smallest possible height thus whole converter fits into $1 \mathrm{U}(40 \mathrm{~mm})$ rack mounted enclosure. It is designed to be powered from three phase $400 \mathrm{~V}_{\mathrm{RMS}}$ and it uses full topology of indirect frequency converter which consist of input LCL filter, AFE and inverter. This topology is capable of: 1) stand-alone function, 2) recuperation of energy through ac-link.

Power stage is build as a standard two-level converter but it uses silicon-carbide $(\mathrm{SiC})$ power switches instead of more common silicon IGBTs. By using SiC components there is possibility to achieve switching frequencies up-to $50 \mathrm{kHz}$ even with limited cooling caused by low height of the converter. Power stage is designed to be able to source current up-to $5 \mathrm{~A}_{\mathrm{RMS}}$.

\section{B. Inputs and outputs}

The main board offers four insulated inputs with $24 \mathrm{~V}$ logic and three outputs with $24 \mathrm{~V} 1 \mathrm{~A}$ capability. There is insulated CAN bus available at the front panel together with insulated digital synchronization signal.

Position or a speed sensor of a motor is connected to simple add-on module which converts signals from sensor to a main control module. It can support both analog ( $\sin -\cos )$ and digital (IRC, ARC) sensor types as it has connection to both analog and digital pins of module. An essential function is also to create insulation barrier between sensor and converter to avoid ground-loops.

\section{Control module}

The frequency converter is controlled by a single board control module called Rice Universal Microcontroller Module (RUMM). Block schematic of the module is shown in Fig. 3 and finished module is in Fig. 4. It is based on latest generation of C2000 core based microcontroller TMS320F28377S and Cyclone III FPGA.

It is designed to be as universal as possible to be able to cover wide range of applications. Signals of the module are provided on three pin headers, one for analog signals, another for digital signals and the last one for optional external EtherCat or similar module. The external module can be connected to SPI or 16-bit parallel bus. Most important RUMM properties are:

- 14 analog bipolar differential inputs divided to four simultaneous sampling groups with 12-bit resolution (up-to 1.1 MSps).

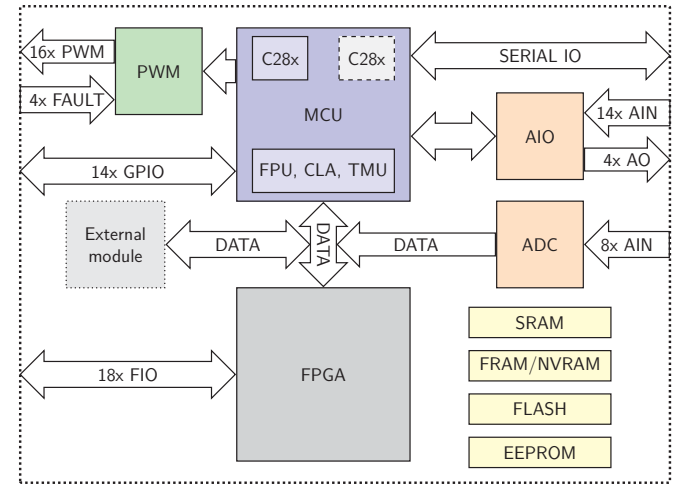

Fig. 3. Control module block schematics

- 8 analog bipolar inputs with simultaneous sampling and 14-bit resolution (up-to $250 \mathrm{kSps}$ ),

- 4 analog outputs with 12-bit resolution, output voltage range $0-2.5 \mathrm{~V}$,

- 16 level-shifted PWMs with output voltage level in range from 5 up to $35 \mathrm{~V}$,

- 4 FAULT inputs with input voltage range up-to $35 \mathrm{~V}$,

- 14 LVTTL GPIOs directly connected to microcontroler,

- 18 LVTTL GPIOs directly connected to FPGA,

- shared SPI bus with 5 chip selects,

- SPI connected dataflash $32 \mathrm{MB}$,

- optional $\mathrm{I}^{2} \mathrm{C}$ with RFID $E^{2} \mathrm{PROM}$,

- optional SRAM with capacity up-to $1 \mathrm{MB}$,

- optional non-volatile SRAM/FRAM up-to $1 \mathrm{MB}$,

- ability to program FPGA configuration FLASH through MCU.

A new generation of digital signal processing enabled microcontroller TMS320F28377S is significantly improved in comparison with older TMS320F28335. It utilizes trigonometric math unit (TMU) which noticeably speeds up calculations of trigonometric functions (sin, cos, sqrt, atan etc.). This unit also adds support for hardware division of single precision float numbers. Moreover user can utilize floating-point co-processor CLA (Command Law Accelerator) which can run in parallel with main core. A computing power of module can be almost doubled by usage of dual-core variant TMS320F28377D of microcontroller (2x MCU core + 2x CLA).

Nowadays, popularity of special communication protocols for absolute position sensors - namely Hiperface [4], EnDat [5], BiSS [6] grows. They replace older SSI and even parallel readings of data from position sensors mainly because they provide bidirectional communication with position sensors thus enable configuring sensor during run-time, they can compensate line delays of cable etc. However they also have some disadvantages: they are usually proprietary (EnDat, Hiperface) and they are hard to implement in a pure microcontroller. Therefore, manufacturers of sensors usually provide locked FPGA entity which covers the whole protocol. This fact leads to usage of a FPGA on RUMM. 


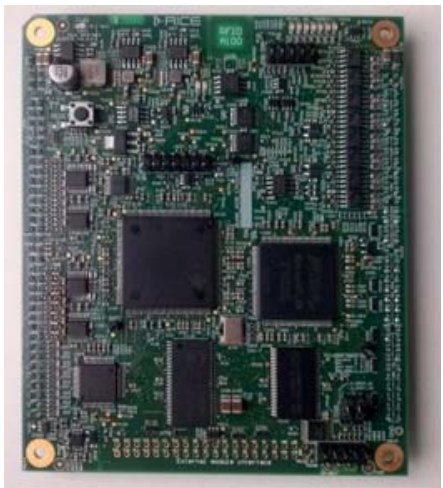

Fig. 4. RUMM module

The FPGA is connected to microcontroller through 16-bit wide data and 9-bit wide address bus (this approach was successfully tested on laboratory development kit [7]). Registers of the FPGA design are memory mapped to address space of the microcontroller. Beside its function as position sensor protocol decoder, the FPGA is responsible for low level blocking of PWM outputs and it provides 18 LVTTL user configurable $\mathrm{I} / \mathrm{O}$ pins on side header of module.

\section{Control Algorithms}

The indirect frequency converter is composed of the voltage source active rectifier (AFE) and voltage source voltage inverter (VSI). The topologies of these converters are the same, the main difference is in their operation modes.

The AFE enables to get nearly sinuoidal current from the grid with zero phase shift between grid phase voltage and phase current (power factor $\lambda \approx 1$ ). For proper function of the AFE the dc-link voltage has to be higher in comparison with standard diode rectifier solution. The dc-link voltage has to be controlled by properly designed control algorithm. The AFE also enables full recuperation mode. The recuperated energy is returned to the grid or it can be utilized by another converter connected in parallel to the first one.

\section{A. Voltage source inverter and motor}

The VSI enables efficient and dynamic control of the LPMSM connected to its output terminals.

The control algorithms for both input and output part have been designed to use maximum of the same components. Algorithms for both parts are based on cascaded PI control structures (vector or FOC control). The basic scheme of the vector control of the linear permanent magnet synchronous motor (LPMSM) is shown in Fig. 5. The main input components for the control algorithms are at least two stator currents $i_{a}, i_{b}$ and rotor position $\vartheta_{m}$. The currents are transformed in a rotating reference frame $d q$. It should be noted that in a standard rotating machine the rotor contains permanent magnets and it rotates and stator is stationary and is composed of three phase winding. In the case of this linear actuator, the moving part is the stator with three phase winding and the stationarv part is the

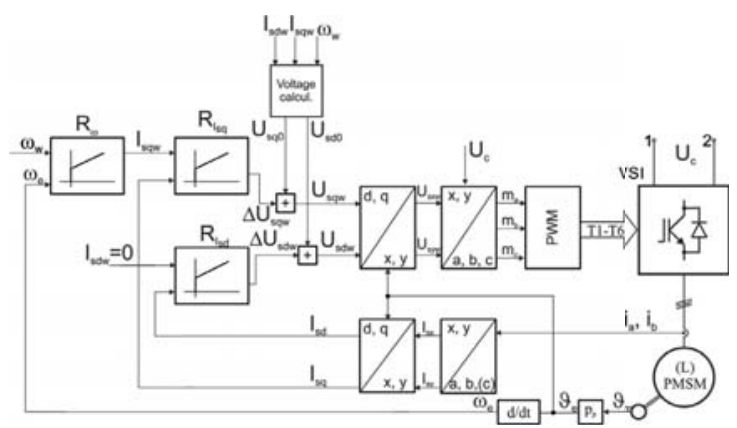

Fig. 5. Block scheme of LPMSM vector control algorithm

rotor with permanent magnets. Terminals 1 and 2 are connected to the AFE dc-link capacitor (Fig. 6).

The current components $I_{s d}$ and $I_{s q}$ are led into current PI controllers. The demanded values $I_{s d w}$ and $I_{s q w}$ represents demanded magnetic flux and force respectively. A flux weakening operation is not assumed in this application, therefore $I_{s d w}=0$. The demanded force which is proportinoal to $I_{s q w}$ is obtained from the speed controller $R_{\omega}$. The desired (servo) application leads to suitable use of a position control. A proportional position controller can be placed ahead of the speed controller. More detailed description of the control algorithm can be found e.g. in [8] with focus on a rotating machine.

\section{B. Voltage source active rectifier}

The basic block scheme of control algorithm of the AFE is shown in Fig. 6. Synchronization of the control algorithms with grid voltages is needed. It is ensured by PLL whose output represents electrical angle of the grid voltage vector $\vartheta_{e}$. This angle is used for transformation of grid currents to the rotating reference frame $d q$ which is in general different from the rotating reference frame of the machine. It is assumed $I_{s d w}=0$ for zero phase shift among grid voltages and currents, it also means that reactive power of the converter is set to zero. Current component $I_{s q w}$ is responsible for delivering of active power to the circuit and to control the dc-link voltage $U_{c}$. The output of the control algorithm is a vector of demanded voltage $U_{v}$ and its position in rotation reference frame $\alpha$. These signals are recalculated to modulation signals and led to the PWM modulator to create switching signals for converter transistors. Further details on control of AFE can be found e.g. in [9].

\section{INITIAL EXPERIMENTS}

First experiments have been performed on the first revision of the converer hardware with rated power of $2 \mathrm{~kW}$ which is shown in Fig. 7.

Very important and difficult operation mode is start up of the AFE. As can be seen from 6, its input part is composed of a three phase LCL filter which represents naturaly almost undamped oscillation circuit. At first, the dc-link is precharged via resistors in the input part. When the dc-link is almost at the rated value around $565 \mathrm{~V}$. the resistors are clamped by relavs. Then the 


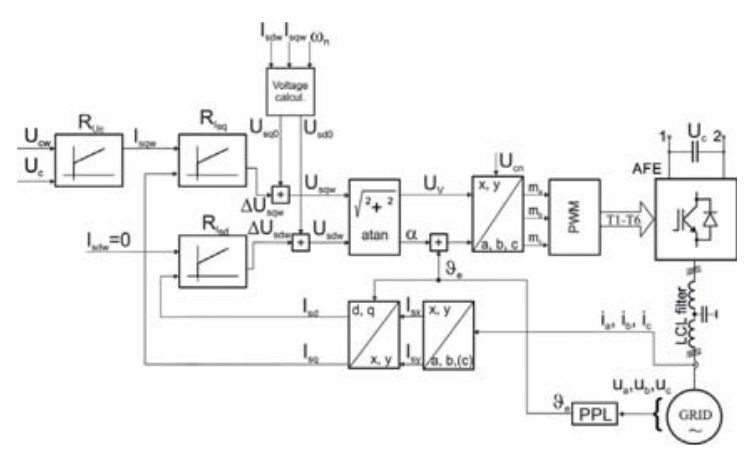

Fig. 6. Block scheme of AFE vector control algorithm

control algorithm starts and ensures the increase of the dc-link voltage upto around $675 \mathrm{~V}$. The start up transient can be seen in Fig. 8 .

Because of very small inductances in the input filter and relatively high dc-link voltage the current ripple in unloaded converter is quite high as can be seen from the blue waveform.

After rectifier star up, it is possible to start the VSI and motor control depending on the superior control system command.

A transient in the inverter circuit with motor speed reversal is shown in Fig. 9. The speed reversal has been performed in range $\pm 16 \mathrm{~Hz}$. Excellent dynamics of the control algorithms can be seen here.

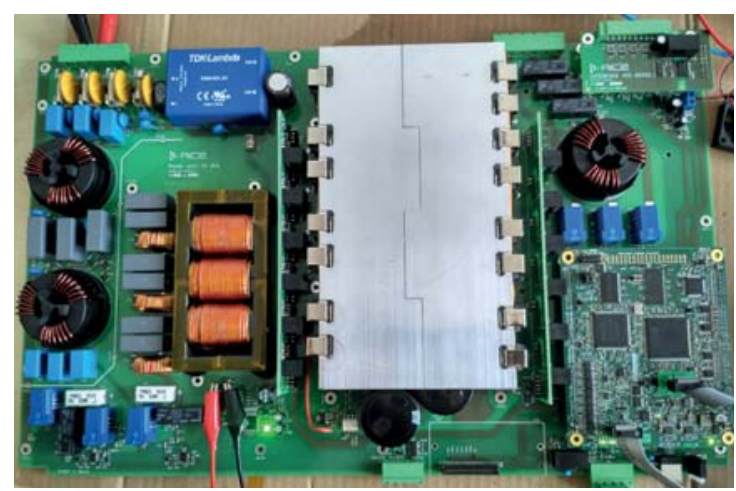

Fig. 7. First revision of converter hardware

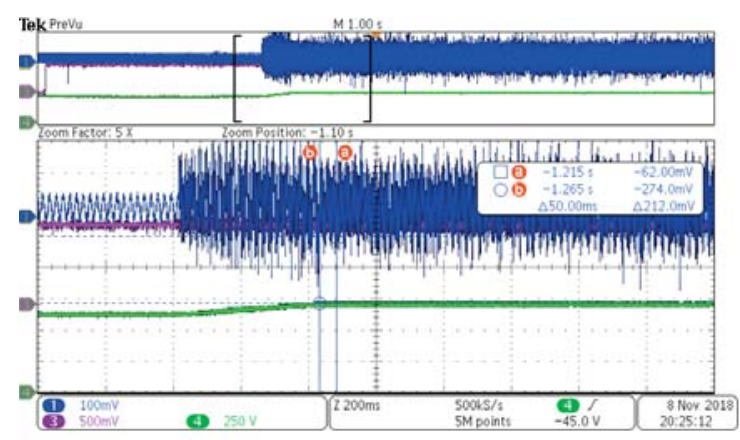

Fig. 8. Experiment:Start up of the active rectifier. Green:dc-link voltage; blue: rectifier input current (100 mV/A).

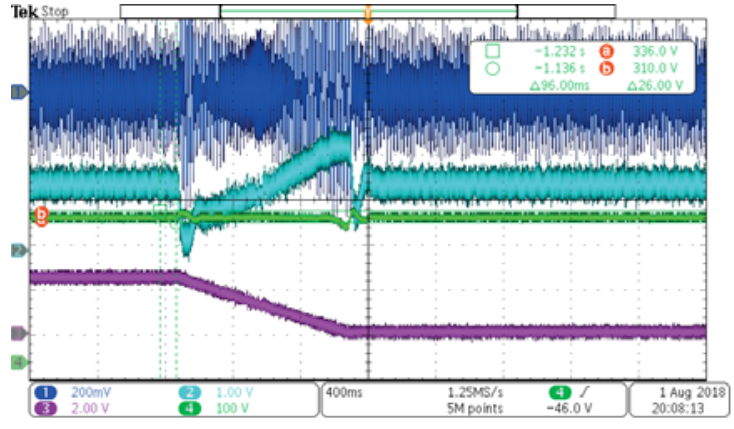

Fig. 9. Experiment:Speed reversal of the motor. Green:dc-link voltage; blue: rectifier input current $(100 \mathrm{mV} / \mathrm{A})$, cyan: rectifier $I_{\text {sqw }}, 3 \mathrm{~A} / \mathrm{div}$, magenta: stator electric frequency $23 \mathrm{~Hz} / \mathrm{div}$.

\section{CONCLUSION}

A new proposal of an indirect frequency converter has been introduced in this paper. The converter is composed as a complete modular unit with the input filter, the active voltage source rectifier and the output voltage source inverter. This configuration enables full energy recuperation. The power hardware is supported by a new control system based on TMS320F28337 which ensures significant computational performance. The new design has been verified by experiments on a laboratory prototype of LPMSM actuator and it shows very high dynamics which is important for the future industrial implementation.

\section{REFERENCES}

[1] J. Li, T. Tang, T. Wang, and G. Yao, "Modeling and simulation for common dc bus multi-motor drive systems based on activity cycle diagrams," in 2010 IEEE International Symposium on Industrial Electronics, July 2010, pp. 250-255.

[2] D. Kumar, F. Zare, and A. Ghosh, "Dc microgrid technology: System architectures, ac grid interfaces, grounding schemes, power quality, communication networks, applications, and standardizations aspects," IEEE Access, vol. 5, pp. 12230-12 256, 2017.

[3] E. Thöndel, "Modelling and simulation of a 6dof motion platform with permanent magnet linear actuators for testing in wind tunnel," in Modelling and Simulation 2014 - European Simulation and Modelling Conference, ESM 2014, 2014, pp. 403-408, cited By :1. [Online]. Available: www.scopus.com

[4] SICK AG, "HIPERFACE DSL® - the digital evolution." [Online]. Available: https://www.sick.com/ag/en/technologies/hiperface-dsl-thedigital-evolution/w/hiperface-dsl/

[5] DR. JOHANNES HEIDENHAIN GmbH, "EnDat 2.2 - The bidirectional interface for encoders." [Online]. Available: https://www.sick.com/ag/en/technologies/hiperfacedsl-the-digital-evolution/w/hiperface-dsl/

[6] iC-Haus GmbH, "BiSS Interface." [Online]. Available: https://www.sick.com/ag/en/technologies/hiperface-dslthe-digital-evolution/w/hiperface-dsl/

[7] T. Kosan, M. Jara, D. Janik, and Z. Peroutka, "Complete development platform for multi-level converters and complex control algorithms," in Mechatronics - Mechatronika (ME), 16th International Conference, Dec 2014, p. pp 152-157.

[8] Z. Peroutka, V. Smidl, and D. Vosmik, "Challenges and limits of extended kalman filter based sensorless control of permanent magnet synchronous machine drives," in 2009 13th European Conference on Power Electronics and Applications, Sep. 2009, pp. $1-11$.

[9] H. Zhang and Y. Zhao, "Vector decoupling controlled pwm rcetifier for wind power grid-connected inverter," in 2009 International Conference on Energy and Environment Technology, vol. 2, Oct 2009, pp. 373-376. 